University of Nebraska - Lincoln

DigitalCommons@University of Nebraska - Lincoln

6-1-1998

\title{
Coercivity and exchange coupling in PrCo:Co nanocomposite
}

\section{films}

J. Ping Liu

University of Nebraska-Lincoln, pliu@uta.edu

Yi Liu

University of Nebraska-Lincoln, yliu@unl.edu

David J. Sellmyer

University of Nebraska-Lincoln, dsellmyer@unl.edu

Follow this and additional works at: https://digitalcommons.unl.edu/physicssellmyer

Part of the Physics Commons

Liu, J. Ping; Liu, Yi; and Sellmyer, David J., "Coercivity and exchange coupling in PrCo:Co nanocomposite films" (1998). David Sellmyer Publications. 81.

https://digitalcommons.unl.edu/physicssellmyer/81

This Article is brought to you for free and open access by the Research Papers in Physics and Astronomy at DigitalCommons@University of Nebraska - Lincoln. It has been accepted for inclusion in David Sellmyer Publications by an authorized administrator of DigitalCommons@University of Nebraska - Lincoln. 


\title{
Coercivity and exchange coupling in PrCo:Co nanocomposite films
}

\author{
J. P. Liu, ${ }^{\text {a),b) }}$ Y. Liu, ${ }^{\text {c) }}$ and D. J. Sellmyer ${ }^{\text {b) }}$ \\ Center for Materials Research and Analysis, University of Nebraska, Lincoln, NE 68588
}

\begin{abstract}
Magnetic hysteresis and intergrain exchange coupling in nanostructured PrCo:Co composite films have been investigated. The composite thin films were made by multilayer sputtering and subsequent annealing. It is found that the coercivity mechanism is related to film morphology, especially the Co phase fraction. Evidence for the hard-soft-phase exchange coupling has been found and high energy products have been obtained. (c) 1998 American Institute of Physics. [S0021-8979(98)26011-7]
\end{abstract}

\section{INTRODUCTION}

Theoretical calculations have predicted giant energy products in exchange-coupled nanostructured hard-softphase composites. ${ }^{1,2}$ However, it is difficult to obtain an ideal nanostructure experimentally. Grain growth during heat treatment and distribution of the second phases cannot be controlled and tailored easily. In addition, it is found that coercivity usually decreases dramatically as the soft phase is added. Among various methods of making nanostructured composites, physical vapor deposition has advantages in controlling grain size and distribution of second phase. We have obtained clear evidence of intergrain exchange coupling and remanence enhancement in $\mathrm{SmCo} / \mathrm{Co}$ multilayer $^{3}$ and FePt-based nanocomposites. ${ }^{4,5}$ In a recent investigation, ${ }^{6}$ we have successfully inserted the soft Co phase into the hard hexagonal PrCo phase thin films to enhance the remanent magnetizations without losing coercivity within a certain thickness of the Co layers. However, the domain structure remained unclear and direct evidence of exchange coupling was not found. In this investigation we study the grain morphology, domain structure, coercivity mechanism, and exchange-coupling behavior in the PrCo:Co nanocomposite films (In this article we use notation $\mathrm{PrCo} / \mathrm{Co}$ to represent the as-deposited multilayers and PrCo:Co the composites after annealing.)

\section{EXPERIMENT}

The $\mathrm{PrCo} / \mathrm{Co}$ multilayer thin films with $\mathrm{Cr}$ underlayer and cover layer were prepared with a multiple-gun dc- and rf-sputtering system by depositing the targets onto glass or silicon substrates. The PrCo target was made by compressing Co and Pr powders with the atomic ratio 7:2 and sintering at $1050{ }^{\circ} \mathrm{C}$ for $1 \mathrm{~h}$. Flowing high-purity argon gas was used for sputtering and the pressures were varied from 5 to 20 mTorr. The sputtering parameters in this investigation (including the

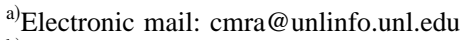

b) Also at: Behlen Laboratory of Physics.

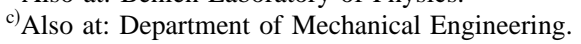

argon pressure, the power rates of sputtering targets, and the distance between the targets and the substrates) were chosen to produce films with maximum coercivity. The thickness of each film was calculated by measuring the mass gain upon deposition. The as-deposited films were then heat treated in a furnace with vacuum $6 \times 10^{-7}$ Torr at temperatures up to $500{ }^{\circ} \mathrm{C}$.

Magnetization loops were measured by an alternating gradient force magnetometer and a superconducting quantum interference device (SQUID) magnetometer. Magnetic force
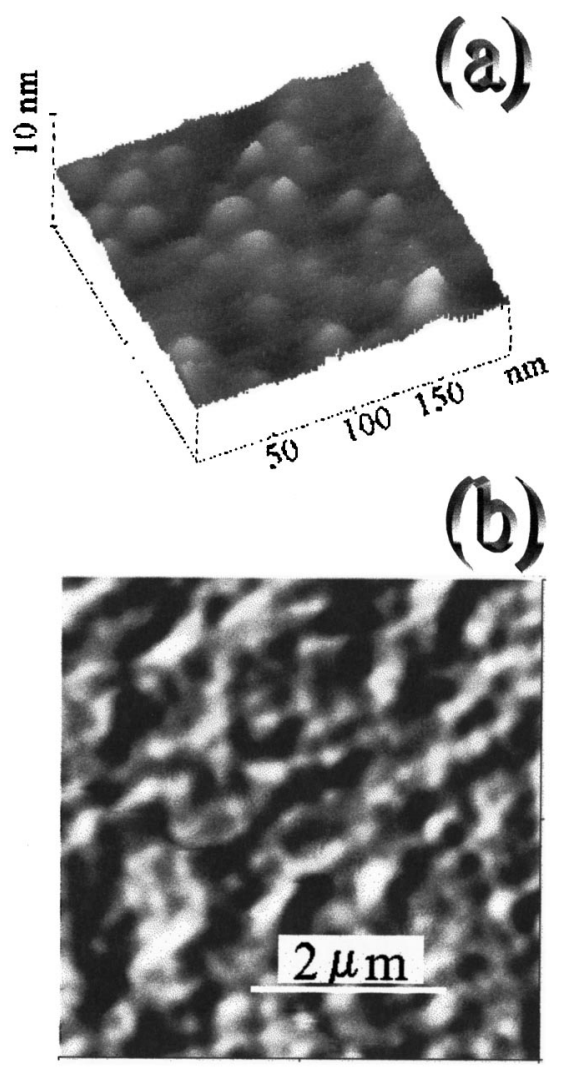

FIG. 1. The AFM (a) and MFM (b) images of a (PrCo30 nm/Co11 nm) $\times 10$ sample with heat treatment at $500{ }^{\circ} \mathrm{C}$. 


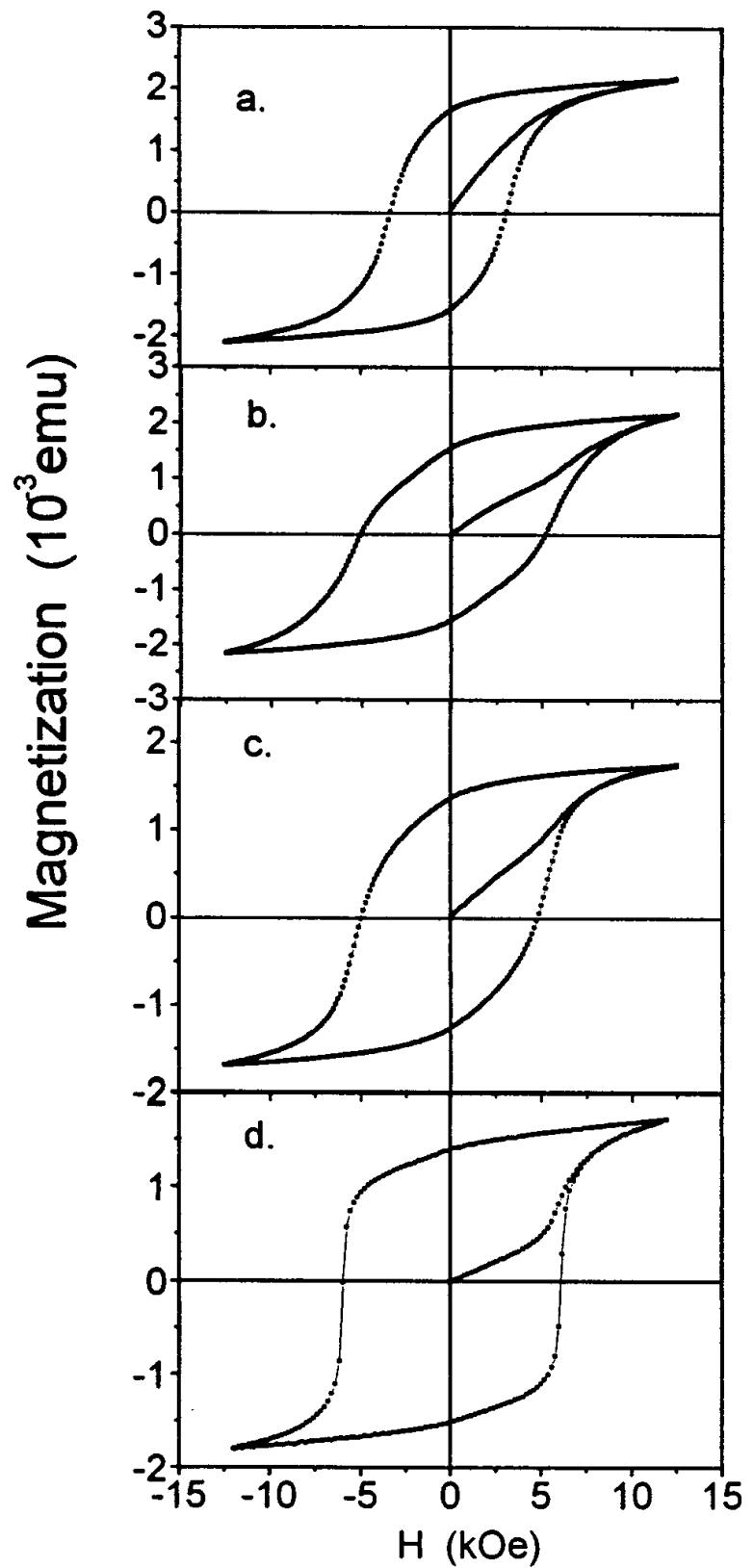

FIG. 2. Hysteresis loops of the heat treated multilyers with structure $(\operatorname{PrCo} 30 \mathrm{~nm} / \mathrm{Co} \mathrm{nm}) \times 10$. In a, $x=11 \mathrm{~nm}$, in b, $x=10 \mathrm{~nm}, \mathrm{c}, x=8 \mathrm{~nm}$, and in $\mathrm{d}, x=5 \mathrm{~nm}$. The magnetization was measured with the field in the film plane.

microscopy (MFM) and atomic force microscopy (AFM) were used to observe the domain structures and the surface morphology. More details about the crystal structure and anisotropy of the films are given in Ref. 6 .

\section{RESULTS AND DISCUSSION}

Figure 1 shows the AFM image of nanostructured PrCo:Co grain morphology (a) and the MFM image of the domain pattern (b). It can be seen that the average size of a domain is about ten times larger than an average grain size (about $20 \mathrm{~nm}$, consistent with our early TEM observation), ${ }^{6}$ indicating that in one domain, many grains are included. As discussed in our previous study, ${ }^{6}$ the PrCo:Co composite consists of the PrCo grains with a disordered hexagonal
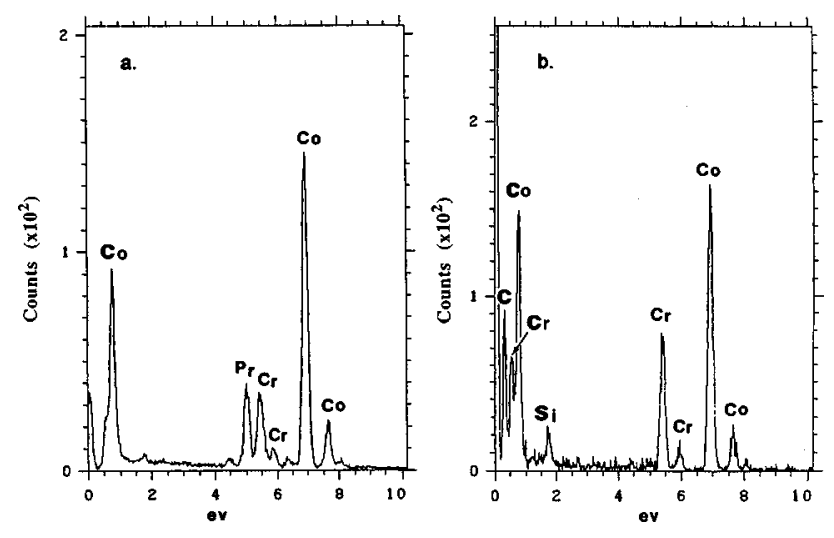

FIG. 3. EDX spectra of a heat treated PrCo:Co film. (a) from the matrix grain and (b) from the second phase grain.

structure and elemental Co with the hexagonal close-packed structure. It is important to note that Co grains have much smaller grain size than the PrCo grains because the Co layers are much thinner than the PrCo layers when the multilayers were sputtered. The grain size we observe in Fig. 1 is basically for the matrix PrCo grains. According to theoretical calculation, ${ }^{2}$ for effective intergrain exchange coupling the dimension of the soft phase should not be bigger than twice that of the domain-wall thickness of the hard phase. In rareearth transition-metal compounds, the typical thickness of domain walls is $3-5 \mathrm{~nm}$. Therefore if the grain size of the soft phase is less than $10 \mathrm{~nm}$, effective intergrain exchange coupling should exist. The grain size of Co phase in the annealed samples can be controlled by controlling the Co layer thickness in the multilayers. This has been demonstrated in our previous experiments. ${ }^{6}$ The critical point is how to deal with the trade-off between coercivity and remanent magnetization as the amount of soft phase is increased. To optimize the energy product the effect of the soft phase on coercivity must be investigated.

Figure 2 shows the initial magnetization curves and the hysteresis loops of a group of samples with decreasing Co layer thicknesses from sample a to sample d. It is interesting to see the change of the initial magnetization curves. For sample a with multilayer structure $(\operatorname{PrCo} 30 \mathrm{~nm} / \mathrm{Co} 11 \mathrm{~nm})$ $\times 10$, the initial curve is similar to that expected in a nucleation-type mechanism of coercivity; whereas for sample $d$ with multilayer structure (PrCo30 nm/Co5 nm) $\times 10$, the initial curve is typical for wall-pinning controlled reversal. However, in case of nanoscale morphology, one may have to consider interaction domains, ${ }^{7,8}$ because simple nucleation and pinning models might not adequate for describing the coercivity mechanism. A study of the details of coercivity mechanism is underway and will be reported later. One point clearly seen from Fig. 2 is that the increase in thickness of the soft Co layers, therefore the Co phase fraction in the annealed samples has changed the magnetizationreversal process.

It is also seen from Fig. 2 that with increasing concentration of Co phase, squareness of the loops become smaller and so does the coercivity. The change in the squareness is associated with a change in the film's morphology and the 


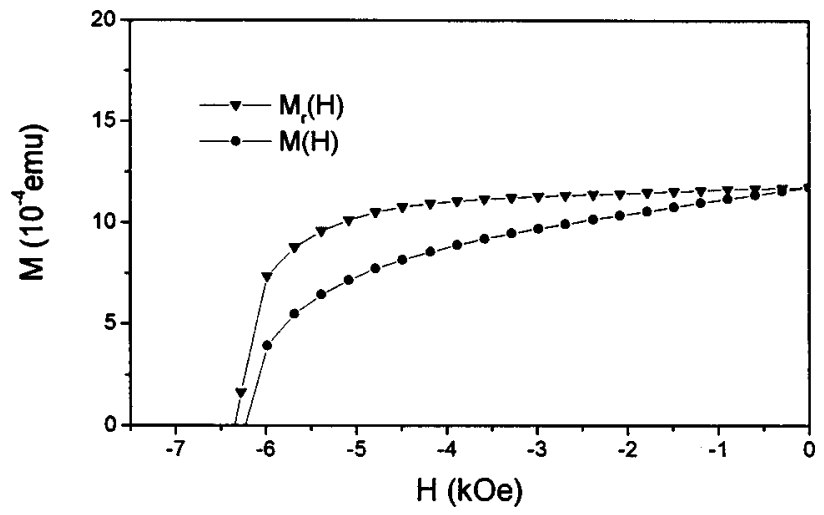

FIG. 4. The demagnetizing curve $M(H)$ and the remanence curve $M_{r}(H)$ of an annealed sample $(\operatorname{PrCo} 30 \mathrm{~nm} / \mathrm{Co} 8 \mathrm{~nm}) \times 10$.

coercivity mechanism. It has been found in our experiment that the squareness and the coercivity is also strongly dependent on heat treatment. In the as-deposited films, the PrCo layer is amorphous which is magnetically soft. Heat treatment produces the magnetically hard hexagonal phase and the coercivity reaches its maximum at heat treatment temperature of $500{ }^{\circ} \mathrm{C}$ for a half-hour annealing. In order to minimize grain growth during the heat treatment, rapid thermal processing has been adopted in this investigation with heating rates up to $100{ }^{\circ} \mathrm{C} / \mathrm{s}$. It is found that a two-step treatment is better than a single-step treatment in obtaining greater squareness and coercivity.

Another important feature observed from Fig. 2 is that in all the cases, the ratio of remanence $M_{r}$ to saturation $M_{s}\left(M_{r} / M_{s}\right)$ is larger than 0.5 . This is an indication that there is intergrain exchange interactions between the grains, as discussed in Ref. 6. To verify that the exchange coupling takes place between the soft phase and the hard phase, we checked the nanostructure by TEM with energy dispersive $\mathrm{x}$-ray (EDX) spectroscopy and by measuring remanence curve to see the exchange-spring behavior. Figure 3 shows the EDX spectra measured in a film in two adjacent grains. One is the matrix grain (a) and another is the soft-phase grain (b). Except for Cr peaks which are from the cover layer and bottom layer and a trace of $\mathrm{Si}$ and $\mathrm{C}$ which may be from the substrate and the contamination during the specimen preparation, in (a) we detected Co and Pr elements, and in (b) we only detected Co. This shows clearly that there are two phases, the hard PrCo phase and soft Co phase existing in the composite. No third phase has been found. Figure 4 shows the remanent magnetization curve $M_{r}(H)$ (also called as dcdemagnetization curve) and the demagnetizing curve $M(H)$ (the part in the second quadrant of the hysteresis loop) of a heat-treated sample. The flatness of the upper curve is equivalent to the behavior Kneller and Hawig ${ }^{1}$ demonstrated for the exchange-spring magnets.

With the understanding of morphology and properties of the $\mathrm{PrCo} / \mathrm{Co}$ composite films discussed above, we have been able to optimize the sputtering and the heat-treatment processes. We added up to $25 \%$ Co into the composite which leads to enhanced remanent magnetization without losing coercivity significantly. By a carefully controlled processing, we have succeeded in obtaining films with coercivity as high as $20 \mathrm{kOe}$ and maximum energy products as high as 25 MGOe at room temperature.

\section{CONCLUSION}

Magnetization behavior of PrCo:Co composite films has been studied including the initial curves and hysteresis loops. The results show that increasing the amount of soft phase appears to change the coercivity mechanism from wallpinning-like to nucleation-like. It is also found that the coercive force and the shape of the loops are very sensitive to heat treatment. Compared with any single-step treatment, a combination of two or more steps in the heat treatment can raise coercivity markedly and improve the squareness of the loops. Optimizing the heat treatment and composite nanostructure leads to high energy products.

\section{ACKNOWLEDGMENT}

We are grateful to Professor S. W. Liou for his help in the MFM and AFM observations. Research sponsored by the U.S. Department of Energy under Grant Nos. DOE-DEF602-86ER45262 and DOE/ANL-95-47DH-007.

${ }^{1}$ E. F. Kneller and R. Hawig, IEEE Trans. Magn. 27, 3588 (1991).

${ }^{2}$ R. Skomski and J. M. D. Coey, Phys. Rev. B 48, 15812 (1993).

${ }^{3}$ I. A. Al-Omari and D. J. Sellmyer, Phys. Rev. B 52, 3441 (1995),

${ }^{4}$ J. P. Liu, Y. Liu, C. P. Luo, and D. J. Sellmyer, J. Appl. Phys. 81, 5644 (1997).

${ }^{5}$ J. P. Liu, C. P. Luo, Y. Liu, and D. J. Sellmyer, Appl. Phys. Lett. (in press).

${ }^{6}$ J. P. Liu, Z. S. Shan, and D. J. Sellmyer, IEEE Trans. Magn. 33, 3709 (1997).

${ }^{7}$ W. Rave, D. Eckert, R. Schäfer, B. Gebel, and K.-H. Miller, IEEE Trans. Magn. 32, 4362 (1996).

${ }^{8}$ R. K. Mishra and R. W. Lee, Appl. Phys. Lett. 48, 733 (1986). 